\title{
Associação entre o perfil de ambiente e condições de trabalho com a percepção de saúde e qualidade de vida em professores de educação básica
}

\section{Association between the environment and work conditions profile and the perception of health and quality of life in teachers in elementary education}

\author{
Érico Felden Pereira ${ }^{1}$, Clarissa Stefani Teixeira² ${ }^{2}$ Rubian Diego Andrade ${ }^{3}$, \\ Fernanda Tolentino de Souza Bleyer ${ }^{4}$, Adair da Silva Lopes ${ }^{5}$
}

\begin{abstract}
Resumo
Objetivo: Analisar as associações entre perfil de ambiente e condições de trabalho com a qualidade de vida de professores. Métodos: Estudo descritivo-transversal com 349 professores de Educação Básica de Florianópolis das redes estadual e municipal de ensino. Os professores responderam ao "Perfil de Ambiente e Condições de Trabalho" e questões de saúde. As variáveis foram associadas por meio de regressão linear, considerando-se como variável dependente a qualidade de vida e como variáveis explicativas a percepção das condições de trabalho. Resultados: As dimensões remuneração e benefícios e ambiente físico foram as que apresentaram maior insatisfação. Mais da metade dos professores estavam insatisfeitos com questões ergonômicas do mobiliário e equipamentos e com as condições de ruído e temperatura; 52,8\% apresentavam esgotamento mental sempre/frequentemente e 50,1\% afirmaram ter dificuldades com alunos agressivos. O perfil de ambiente e das condições de trabalho explicou 24,6\% da avaliação do domínio meio ambiente de qualidade de vida. Conclusão: Os professores percebem que os benefícios da carreira estão aquém da relevância e das demandas da profissão. A insatisfação com as condições de trabalho se materializa pela diminuição da saúde desses profissionais. Além disso, o apoio social no ambiente escolar parece tornar as condições de ambiente mais favorável ao trabalho.

Palavras-chave: saúde escolar; saúde do trabalhador; desenvolvimento humano.
\end{abstract}

\begin{abstract}
Objective: To analyze the associations between environment and work conditions profile and teachers' quality of life. Methods: Descriptivecross-sectional study with 349 teachers of Basic Education from Florianópolis, Santa Catarina, Brazil, regarding state and municipal systems. The teachers answered the "Environment and Work Conditions Profile" and health questions. The variables were related by linear regression considering the quality of life as dependent variable and the perception of working conditions as explanatory variable. Results: The dimensions compensation and benefits and physical environment showed high percentage of dissatisfaction. More than half of the teachers were dissatisfied with the ergonomic furniture and equipment and noisy and temperature conditions; $52.8 \%$ presented mental exhaustion always/often and $50.1 \%$ reported difficulties due to aggressive students. Environment and work conditions profile explained $24.6 \%$ of environment domain of quality of life evaluation. Conclusion: Teachers perceive that the career benefits are below the relevance and the demands of the profession. Dissatisfaction with working conditions is materialized by the reduction of these professionals' health. Furthermore, social support in the school environment seems to make conditions more favorable to the work. Keywords: school health; occupational health; human development.
\end{abstract}

Trabalho realizado na Universidade do Estado de Santa Catarina (UDESC) - Florianópolis (SC), Brasil.

'Doutor em Educação Física; Professor Adjunto de Aprendizagem Motora e Desenvolvimento Humano do Centro de Ciências da Saúde e do Esporte da UDESC Florianópolis (SC), Brasil.

2Doutora em Engenharia de Produção; Professora da Sociedade Educacional de Santa Catarina (SOCIESC) - Florianópolis (SC), Brasil.

${ }^{3}$ Mestrando em Ciências do Movimento Humano do Centro de Ciências da Saúde e do Esporte da UDESC - Florianópolis (SC), Brasil.

${ }^{4}$ Mestranda em Ciências do Movimento Humano; Professora do Centro de Ciências da Saúde e do Esporte da UDESC - Florianópolis (SC), Brasil.

${ }^{5}$ Doutor em Ciência do Movimento Humano; Professor Titular do Centro de Desportos da Universidade Federal de Santa Catarina (UFSC) - Florianópolis (SC), Brasil.

Endereço para correspondência: Érico Felden Pereira - CEFID/UDESC - Rua Paschoal Simoni, 358 - CEP: 88080-350 - Florianópolis (SC), Brasil -

E-mail: ericofelden@gmail.com

Fonte de financiamento: nenhuma.

Conflito de interesses: nada a declarar. 


\section{INTRODUÇÃO}

A precarização do trabalho docente faz parte de um processo histórico complexo, cujos reflexos na qualidade do ensino e na saúde dos professores vêm se tornando cada vez mais evidentes. As relações entre a condição de saúde e o trabalho têm sido investigadas por diferentes áreas do conhecimento, revelando a preocupação dos pesquisadores ao se considerarem, por exemplo, os crescentes índices de professores que desistem do magistério em função de condições degradantes de seu trabalho ${ }^{1}$.

Nesse contexto, o estudo coordenado por Codo ${ }^{2}$ foi pioneiro e proporcionou maior visibilidade a situações sentidas e vividas diariamente na prática docente, mostrando que, aproximadamente $70 \%$ dos profissionais de educação no Brasil apresentavam indicativos de exaustão emocional.

Esse quadro é ilustrado também em realidade estrangeiras, como apresentado em recente estudo de acompanhamento conduzido por Ervasti et al. ${ }^{3}$ com professores finlandeses. Neste estudo, identificaram-se crescentes frequências de absenteísmo, e tanto as características e a qualidade do ambiente na escola quanto os problemas psicossociais nos alunos, como, por exemplo, maior presença de bullying, aumentaram significativamente os pedidos de afastamentos do trabalho.

De acordo com dados apresentados por Batista et al. ${ }^{4}$, mais da metade dos afastamentos do trabalho dos professores de educação básica possuem como causa quadros depressivos e, dessa forma, faz-se necessário um olhar especial para a compreensão do adoecimento nessa categoria profissional. Conforme contextualização de Dias et al. ${ }^{5}$, nas últimas décadas, é possível verificar propostas de reformas das políticas educacionais, bem como a incorporação das classes mais populares na escola. Esse processo desencadeia uma série de novas demandas que devem ser atendidas pelos professores, os quais precisam modificar suas práticas e forma de encarar o processo de ensino/ aprendizagem. Esse processo, em conjunto com a formação deficitária, considerando-se a realidade escolar, as más condições de trabalho, o desinteresse dos alunos e de suas famílias na formação e a crescente agressividade dos alunos, agravam o quadro de adoecimento dos professores.

De fato, a profissão docente parece estar especialmente associada com inúmeras alterações na saúde dos professores, sendo que, conforme abordam Souza e Leite ${ }^{6}$, muitas doenças ocupacionais geradas pelas condições de trabalho são mascaradas por doenças comuns, recaindo o ônus nos próprios professores. Assim, o conhecimento mais detalhado da influência dos diferentes aspectos relacionados com as condições do trabalho docente quanto à saúde e qualidade de vida dos professores é relevante, e essa é a proposta deste artigo.

Dessa forma, a qualidade de vida e as condições de trabalho de professores de educação básica ainda são desconhecidas em muitas realidades brasileiras. Em Florianópolis (SC), capital brasileira com melhor Índice de Desenvolvimento Humano $(0,875)$, de acordo com os dados do Programa das Nações Unidas para o Desenvolvimento ${ }^{7}$, nenhum estudo sobre saúde e qualidade de vida de professores de educação básica, especialmente com abrangência populacional, foi encontrado até a realização desta pesquisa.

Objetivou-se, desta forma, analisar algumas associações entre a percepção das condições de trabalho (percepção de ambiente físico, ambiente social, desenvolvimento e realização profissional, remuneração e benefícios e relevância social do trabalho) com a qualidade de vida de professores de educação básica de Florianópolis.

\section{MÉTODOS}

Este estudo caracteriza-se como descritivo-exploratório, de corte transversal, no qual foi realizado um levantamento de informações ainda pouco investigadas em uma determinada população.

\section{Amostra}

A amostra foi formada por 349 professores de educação básica de escolas públicas (estaduais e municipais) de Florianópolis, estimada em 3.188 professores (1.943 da rede estadual e 1.245 da rede municipal), de acordo com dados da Secretaria de Estado da Educação, Ciências e Tecnologia de Santa Catarina e da Secretaria Municipal de Educação.

Com o objetivo de obter uma maior representatividade do município, foram selecionadas, de forma aleatória e proporcional, considerando-se as duas redes de ensino investigadas, escolas de todas as regiões de acordo com a divisão geográfica adotada pela prefeitura municipal de Florianópolis: parte insular, parte continental e periferia. Ao final, foram investigados professores de 18 escolas, distribuídas de forma proporcional nas 3 regiões geográficas citadas.

O número mínimo de professores para a formação da amostra foi calculado pela equação de Rodrigues ${ }^{8}$, considerandose os dados fornecidos pelas secretarias de educação. Assim, o número mínimo de professores calculado foi de 342 . Ao todo, 355 professores responderam à pesquisa, sendo que os questionários de 6 deles foram excluídos em função de preenchimento inadequado.

O estudo foi autorizado pelas secretarias de educação e, posteriormente, pelas direções das escolas. O projeto de pesquisa foi aprovado pelo Comitê de Ética em Pesquisa com Seres Humanos da Universidade Federal de Santa Catarina, protocolado sob número 072/2007, e os professores assinaram o Termo de Consentimento Livre e Esclarecido. 


\section{Indicadores avaliados}

Os professores foram convidados a responder um questionário sobre sua percepção de ambiente e condições de trabalho e percepção de qualidade de vida. Para identificação da percepção dos professores a respeito do seu ambiente de trabalho, foi utilizado o Perfil de Ambiente e Condições de Trabalho9 ${ }^{9}$, formado por 15 questões dentro de uma escala de 4 níveis (1: ruim, 2: regular/sofrível, 3: bom/boa e 4: excelente). O instrumento busca avaliar o ambiente e as condições de trabalho, considerando-se cinco domínios, a saber: ambiente físico; ambiente social; desenvolvimento e realização profissional; remuneração e benefícios; relevância social do trabalho. Neste estudo, as respostas "ruim" e "regular" foram consideradas como indicativo de percepção negativa; as respostas "boa" e "excelente", de percepção positiva.

Para avaliação da percepção de qualidade de vida dos professores, foi aplicado o WHOQOL-bref. Trata-se de um instrumento especialmente adaptado a estudos de cunho epidemiológico, permitindo a comparação entre grupos, comunidades e países ${ }^{10} \mathrm{O}$ WHOQOL-bref foi validado no Brasil ${ }^{11}$ e é composto por 2 questões gerais sobre a satisfação com a saúde e com a qualidade de vida e por outras 24 correspondentes aos domínios físico, psicológico, relações sociais e meio ambiente, sendo cada uma formada por facetas específicas. Os escores da qualidade de vida são expressos de 0 a 100, considerando-se escores superiores como indicativos de percepção mais positiva de qualidade de vida. $\mathrm{O}$ instrumento considera informações das duas últimas semanas dos avaliados e não possui um ponto de corte para classificação da qualidade de vida.

Considerando-se que, tanto o WHOQOL-bref quanto o Perfil de Ambiente e Condições de Trabalho, não são instrumentos específicos para a aplicação em professores, foram formuladas duas questões complementares relacionadas, de forma mais direta, ao ambiente escolar. Com objetivo de melhor entendimento, buscou-se manter uma similaridade dessas questões com as demais do Perfil de Ambiente e Condições de Trabalho. Para essas questões, foi utilizada a seguinte escala: 0 indicando "nunca"; 1 , "raramente"; 2 , "constantemente" e 3 , "sempre”. Foi indicado que essas respostas deveriam ser respondidas considerando-se sua realidade de trabalho na escola. As questões complementares foram avaliadas com relação ao seu conteúdo por cinco especialistas doutores que forneceram sugestões para a versão final aplicada aos professores. As questões complementares foram:

1. consequências do trabalho para a saúde e qualidade de vida:

- esgotamento físico e mental;

- problemas físicos (dores no corpo, problemas com a voz, alergias etc.);

- influência negativa do trabalho na minha saúde/qualidade de vida;
2. condições didático-pedagógicas do trabalho:

- dificuldades com o trabalho em função de alunos agressivos e indisciplinados;

- dificuldade com o trabalho em função da estrutura e segurança da escola;

- dificuldades com o trabalho em função da carga horária.

\section{Análise dos dados}

Além das análises específicas de cada instrumento, foram realizadas medidas descritivas. As associações entre a qualidade de vida e a percepção de ambiente e condições de trabalho foram analisadas utilizando-se análises de regressão linear múltipla, considerando-se como variáveis dependentes os domínios de qualidade de vida e como independente a percepção das condições de trabalho. As análises residuais dos modelos ajustados foram realizadas por meio do teste de Kolmogorov-Smirnov. Optou-se pela análise de associação por meio de regressão linear, já que os instrumentos utilizados não apresentam pontos de corte para classificação e os dados apresentam os pressupostos matemáticos para tal análise. Foi considerado um nível de significância de 5\%.

\section{RESULTADOS}

A amostra teve média de idade de 40,11 $(9,1)$ anos e foi formada, em sua maioria, por mulheres (77,3\%). Verificando-se os resultados apresentados na Tabela 1 , dentre os domínios relacionados à qualidade de vida, "relações sociais" foi o que se aproximou mais do escore 100, sugerindo, assim, uma percepção

Tabela 1. Resultados descritivos das variáveis investigadas

\begin{tabular}{lc} 
Variáveis & Média (DP) \\
Idade (anos) & $40,11(9,1)$ \\
Mulheres (\%) & 77,3 \\
Qualidade de vida (pontos)* & \\
$\quad$ Geral & $63,75(19,16)$ \\
Físico & $65,70(15,39)$ \\
Psicológico & $68,61(13,48)$ \\
Relações sociais & $73,10(17,22)$ \\
Meio ambiente & $53,93(15,05)$ \\
Ambiente de trabalho (pontos) & \\
$\quad$ Ambiente físico & $4,23(1,96)$ \\
$\quad$ Ambiente social & $6,71(1,56)$ \\
$\quad$ Desenvolvimento e realização profissional & $5,43(1,57)$ \\
Remuneração e benefícios & $2,96(1,66)$ \\
Relevância social do trabalho & $5,66(1,42)$ \\
Trabalho docente (pontos) & \\
Consequências do trabalho para a saúde e & \\
qualidade de vida & $4,38(2,06)$ \\
Condições didático-pedagógicas do trabalho & $5,01(2,16)$ \\
\hline Os valores são expressos em média (desvio padrão) ou percentual; \\
*pontuação da escala de qualidade de vida de 0 a 100; * pontuação da \\
escala de Perfil de Ambiente e Condições de Trabalho de 0 a 9.
\end{tabular}


mais positiva, com 73,10 $(17,22)$ média/pontos; o domínio "meio ambiente", entretanto, obteve escore mais baixo, indicando percepção mais negativa.

Considerando-se as questões de ambiente e condições de trabalho, dentre os seus domínios, o "ambiente social" foi o

Tabela 2. Frequências de professores com percepção negativa de acordo com as questões Perfil de Ambiente e Condições de Trabalho

\section{Dimensões e variáveis}

Ambiente físico

Condições de limpeza e iluminação do

local de trabalho

Adequação ergonômica do mobiliário e equipamentos

Condição de ruído e temperatura

Ambiente social

Relacionamento com os demais

trabalhadores

Relacionamento com o(s) chefe(s)

imediatos(s)

Oportunidade para expressar opiniões

relacionadas ao trabalho

Desenvolvimento e realização profissional Oportunidades de crescimento e aperfeiçoamento profissional oferecidos pela instituição

Nível de conhecimento/habilidade para realizar as tarefas

Grau de motivação e ânimo ao chegar para trabalhar

Remuneração e benefícios

Remuneração em relação ao trabalho realizado

Benefícios de saúde oferecidos pela instituição aos trabalhadores

Oportunidades de lazer e congraçamento entre trabalhadores e familiares

Relevância social do trabalho Imagem da instituição perante a sociedade Relevância do trabalho para a instituição e a sociedade

Nível de equilíbrio entre sua vida profissional e pessoal/familiar

Consequências do trabalho para a saúde e qualidade de vida

Esgotamento físico e mental

Problemas físicos (dores no corpo, problemas com a voz, alergias, etc.) Influência negativa do trabalho na saúde/qualidade de vida

Condições didático-pedagógicas do trabalho

Dificuldades com o trabalho em função de alunos agressivos e indisciplinados Dificuldades com o trabalho em função da estrutura e segurança da escola Dificuldades com o trabalho em função da carga horária
Professores com percepção negativa (\%) componente com percepção mais positiva, ao passo que o componente relacionado com a "remuneração e benefício" foi o que apresentou percepção mais negativa (Tabela 1).

$\mathrm{Na}$ Tabela 2, foram apresentados os percentuais de professores com percepção negativa do ambiente de trabalho dentre todas as questões listadas nas dimensões. As questões com maior percentual de professores insatisfeitos foram: remuneração em relação ao trabalho realizado (78,5\%); oportunidades de lazer e congraçamento entre trabalhadores e familiares (73,0\%) e benefícios de saúde oferecidos pela instituição aos trabalhadores (69,3\%). Já as questões com menor percentual de professores insatisfeitos foram: nível de conhecimento/habilidade para realizar as tarefas $(3,4 \%)$; relacionamento com os demais trabalhadores $(6,3 \%)$ e relacionamento com o(s) chefe(s) imediato(s) (9,2\%).

Os dados indicaram, ainda, que mais da metade dos professores estavam insatisfeitos com questões ergonômicas e de ruído e temperatura na escola, assim como com relação às oportunidades de crescimento e aperfeiçoamento profissional oferecidas pela instituição. Por outro lado, o relacionamento com colegas e chefes imediatos mostrou-se adequado para a maioria dos professores.

$\mathrm{Na}$ Tabela 3, foram apresentadas as análises de associação entre qualidade de vida e percepção de ambiente de trabalho. O domínio meio ambiente apresentou maior possibilidade de ser explicado pelas condições de trabalho $(24,6 \%)$ e o ambiente físico, a remuneração e benefícios e a relevância social do trabalho foram as dimensões mais importantes nessa associação. As questões relacionadas com a remuneração e benefícios estiveram significativamente associadas com a qualidade de vida em todos os domínios.

$\mathrm{Na}$ Tabela 4, foram apresentados os resultados das duas questões complementares específicas para professores, na qual se observa que a maioria apresentou percepção negativa, considerando-se variáveis "esgotamento físico e mental" e "problemas físicos", ambos acima de 50\%. Ainda, 40,2\% afirmaram que o trabalho traz influências negativas para sua qualidade de vida.

Na dimensão "Condições didático-pedagógicas do trabalho", 50,1\% dos professores se referiram a dificuldades com o trabalho em função de alunos agressivos e indisciplinados.

\section{DISCUSSÃO}

Neste estudo, investigaram-se algumas associações entre a percepção das condições de trabalho com a qualidade de vida de uma amostra representativa dos professores de educação básica de Florianópolis. Os estudos com professores de Educação Básica são recentes no Brasil e convergem ao mostrarem que se trata de uma categoria profissional cada vez mais exposta a 
Tabela 3. Associação entre a qualidade de vida com o ambiente e condições de trabalho

\begin{tabular}{|c|c|c|c|}
\hline Domínios da qualidade de vida & $\beta$ & Valor $\mathrm{p}^{*}$ & $\mathbf{r}^{2 * *}$ \\
\hline \multicolumn{4}{|l|}{ Qualidade de vida geral } \\
\hline Constante $=50,089$ & & $<0,001$ & $11,0 \%$ \\
\hline Ambiente físico & 0,232 & $<0,001$ & \\
\hline Ambiente social & $-0,112$ & 0,066 & \\
\hline Desenvolvimento e realização profissional & 0,147 & 0,032 & \\
\hline Remuneração e benefícios & $-0,123$ & 0,027 & \\
\hline Relevância social do trabalho & 0,101 & 0,128 & \\
\hline \multicolumn{4}{|l|}{ Domínio físico } \\
\hline Constante $=43,906$ & & $<0,001$ & $18,7 \%$ \\
\hline Ambiente físico & 0,178 & 0,003 & \\
\hline Ambiente social & $-0,053$ & 0,358 & \\
\hline Desenvolvimento e realização profissional & 0,254 & $<0,001$ & \\
\hline Remuneração e benefícios & $-0,161$ & 0,003 & \\
\hline Relevância social do trabalho & 0,167 & 0,009 & \\
\hline \multicolumn{4}{|l|}{ Domínio psicológico } \\
\hline Constante $=47,417$ & & $<0,001$ & $16,8 \%$ \\
\hline Ambiente físico & 0,144 & 0,016 & \\
\hline Ambiente social & 0,053 & 0,366 & \\
\hline Desenvolvimento e realização profissional & 0,020 & 0,769 & \\
\hline Remuneração e benefícios & $-0,154$ & 0,005 & \\
\hline Relevância social do trabalho & 0,344 & $<0,001$ & \\
\hline \multicolumn{4}{|l|}{ Domínio relações sociais } \\
\hline Constante $=49,463$ & & $<0,001$ & $10,0 \%$ \\
\hline Ambiente físico & 0,084 & 0,173 & \\
\hline Ambiente social & 0,169 & 0,006 & \\
\hline Desenvolvimento e realização profissional & 0,032 & 0,648 & \\
\hline Remuneração e benefícios & $-0,142$ & 0,012 & \\
\hline Relevância social do trabalho & 0,152 & 0,023 & \\
\hline \multicolumn{4}{|l|}{ Domínio meio ambiente } \\
\hline Constante $=23,902$ & & $<0,001$ & $24,6 \%$ \\
\hline Ambiente físico & 0,193 & 0,001 & \\
\hline Ambiente social & 0,041 & 0,460 & \\
\hline Desenvolvimento e realização profissional & 0,026 & 0,685 & \\
\hline Remuneração e benefícios & 0,101 & 0,050 & \\
\hline Relevância social do trabalho & 0,284 & $<0,001$ & \\
\hline
\end{tabular}

${ }^{\star}$ Valor $p$ da análise de regressão linear; ${ }^{* *}$ capacidade explicativa dos modelos ajustados.

riscos de inúmeras doenças. É perceptível também nos estudos já realizados a intrincada relação entre o adoecimento docente e as condições de trabalho na escola, bem como com as novas demandas da Educação Básica no Brasil. Destaca-se, nesse contexto, a inexistência de levantamentos a respeito da saúde e condições de trabalho em professores de Educação Básica de Florianópolis até a realização desta pesquisa.

De forma geral, os dados levantados mostram que as questões relacionadas a "remuneração e benefícios", "condição de ruído e temperatura" e a questão "crescimento e aperfeiçoamento profissional oferecidos pela instituição" foram as que apresentaram maiores percentuais de professores insatisfeitos. Por outro lado, a maior parte dos professores revelou que as relações com colegas e chefes são boas, e isso pode estar
Tabela 4. Questões complementares relacionadas à saúde e atuação dos professores na escola

Dimensões e variáveis

Professores com percepção negativa (\%)

Consequências do trabalho para a saúde e qualidade de vida

Esgotamento físico e mental 52,8

Problemas físicos (dores no corpo, $\quad 52,5$

problemas com a voz, alergias etc.)

Influência negativa do trabalho na $\quad 40,2$

saúde/qualidade de vida

Condições didático-pedagógicas do trabalho

Dificuldades com o trabalho em função

de alunos agressivos e indisciplinados

Dificuldades com o trabalho em função $\quad 35,8$

da estrutura e segurança da escola

Dificuldades com o trabalho em função

da carga horária

associado com maior suporte no ambiente do trabalho e ter contribuído para que os professores apresentassem maior pontuação no domínio de relações sociais. Os resultados das análises das condições de trabalho e insatisfação dos professores com aspectos ergonômicos no ambiente escolar encontrados em Florianópolis vão ao encontro dos achados de Penteado e Pereira $^{12}$, que identificaram, em professores de Educação Básica de Rio Claro (SP), que as oportunidades de lazer, condições financeiras, ambiente de trabalho e acesso à informação são situações de maior insatisfação.

Nesse contexto, Nardi e Schneider ${ }^{13}$ esclarecem que, na análise do trabalho docente, faz-se necessário considerar as características inerentes a essa atividade, incluindo-se as condições materiais de trabalho, bem como as políticas institucionais que o regulam. No caso dos professores de Florianópolis, tal consideração é confirmada, indicando-se que a melhoria da percepção da condições de trabalho deve abranger ações governamentais que reflitam no dia-a-dia da escola, como a melhoria nos salários e das condições físicas da escola, bem como a organização das rotinas com vistas às atividades de formação continuada.

O estudo publicado por Alves e Pinto ${ }^{14}$ a partir de dados da Pesquisa Nacional por Amostra de Domicílios e do censo Escolar é esclarecedor da situação financeira dos professores de Educação Básica no Brasil ao apontar, além de dificuldades com carga excessiva de trabalho e desvalorização da profissão, que os professores possuem rendimentos abaixo dos obtidos por outros profissionais com formação equivalente. Assim, já é perceptível uma diminuição na procura pelas licenciaturas pelos jovens, sendo necessários programas governamentais, como, por exemplo, o aumento das vagas em cursos à distância para a formação de professores. Além disso, é possível refletir que a desvalorização salarial afaste os profissionais mais qualificados da atuação docente na Educação Básica e que uma 
remuneração inadequada pode gerar ainda mais volume de trabalho, visto que muitos professores passam a assumir maior carga horária e trabalho em várias escolas, prejudicando ainda mais sua saúde e criando um círculo vicioso.

A má remuneração dos professores também foi relatada no estudo de Marchiori et al. ${ }^{15}$ como um fator importante na análise das condições de trabalho. Neste estudo, as condições de saúde de professores foram investigadas no município de Vitória, com 607 docentes da rede pública. Constatou-se que, além dos baixos salários, outros fatores contribuem para a tensão e cansaço, como o ritmo intenso de trabalho e o número excessivo de alunos em sala de aula.

Nesse sentido, conforme discutem Bauer et al. ${ }^{16}$, uma demanda cada vez mais presente nas escolas e que parece influenciar de forma significativa a saúde dos professores refere-se ao comportamento agressivo dos alunos. O percentual de professores que relataram dificuldades com o trabalho em função de alunos agressivos e indisciplinados nas escolas de Florianópolis foi de $50,1 \%$. Maring e Koblinsky ${ }^{17}$ sugerem como intervenções para melhorar a segurança das escolas a formação de professores em gestão do comportamento, liderança escolar eficaz, a melhoria da segurança escolar, expansão dos serviços de saúde mental e envolvimento dos pais.

Da mesma forma que o relacionamento com colegas pode ter contribuído para que os professores tenham apresentado percepção mais positiva no domínio relações sociais, as questões ergonômicas na escola podem ter sido relevantes para que o domínio meio ambiente apresentasse a menor pontuação do WHOQOL-bref. Diante desses resultados, a organização dos espaços da escola e de atividades sociais que possam fortalecer os vínculos afetivos entre os profissionais de educação deve ser pensada, considerando-se as rotinas de trabalho e o tempo de permanência na escola, incluindo o tempo disponível entre as aulas.

Os resultados identificaram que as condições físicas do ambiente precisam de melhorias, principalmente em relação a ruído e adequações ergonômicas das escolas. Questões como as condições de ruído e temperatura também já vêm sendo investigadas por alguns estudos com professores realizados no Brasil, principalmente relacionados à área da fonoaudiologia ${ }^{12,18,19}$.
Nesse contexto, ambientes ruidosos, grande carga horária, grande número de alunos, entre outros aspectos, estão diretamente relacionados às disfonias e à baixa qualidade de vida dos educadores. Além disso, todas essas características de más condições de trabalho expõem os trabalhadores a fatores estressantes que, se persistentes, podem levá-lo a desenvolver a síndrome de burnout ${ }^{20}$.

Em recente estudo longitudinal realizado com 816 professores na Suécia ${ }^{21}$, os autores constataram que, ao longo dos 3 primeiros anos de carreira, houve um aumento nos casos de burnout, atingindo um nível máximo no terceiro ano de trabalho. Ainda segundo os autores ${ }^{21}$, as variáveis que podem estar associadas são: altos níveis de depressão e baixos níveis de autopercepção de saúde e satisfação com a vida. Estratégias de prevenção de doenças, como a possibilidade de exames periódicos e educação para a saúde, devem ser implementadas desde o início da carreira docente, sendo necessário organizar e acompanhar a adaptação dos professores quando da sua chegada na escola. Assim, uma equipe de apoio pedagógico que conheça e compreenda as reais demandas dos professores de sala de aula pode ser sugerida.

Os resultados do estudo indicaram que as principais medidas para melhoria das condições de trabalho dos professores investigados estão relacionadas com o investimento na valorização salarial e na melhoria do ambiente e equipamentos de trabalho. $\mathrm{O}$ apoio social no ambiente escolar parece tornar as condições de ambiente mais favoráveis ao trabalho, devendo ser promovido. Intervenções que considerem a melhoria das condições de ruído e temperatura, bem como a indisciplina dos alunos, são necessárias. Sugere-se também melhores benefícios, como plano de saúde e odontológico, além de políticas de lazer que contemple os trabalhadores e familiares para uma maior longevidade na carreira docente e melhoria das condições de trabalho dos professores.

Algumas limitações apontadas neste estudo se referem à forma de análise transversal, que limita a discussão de causa e efeito, e ao fato de a coleta ter sido realizada apenas em escolas públicas, sendo necessário o uso de instrumentos mais abrangentes para a análise da saúde na escola.

\section{REFERÊNCIAS}

1. Cruz RM, Lemos JC. Atividade docente, condições de trabalho e processos de saúde. Motrivivência. 2005;24(17):59-80.

2. Codo W. Educação: carinho e trabalho. 2a edição. Petrópolis (RJ): Vozes; 1999.

3. Ervasti J, kivimäki M, Kawachi I, Subramanian SV, Pentti J, Oksanen T, et al. School environment as predictor of teacher sick leave data-linked prospective cohort study. BMC Public Health. $2012 ; 12: 770$
4. Batista JBV, Carlotto MS, Moreira AM. Depressão como causa de afastamento do trabalho: um estudo com professores do ensino fundamental. Psico. 2013;44:2.

5. Dias DDS, Barros MED, Silva OIV, Campos GM. Das dores e delícias do trabalho docente: uma análise do trabalho educação. 2013,22(2):169-81.

6. Souza NA, Leite MP. Condições de trabalho e suas repercussões na saúde dos professores da educação básica no Brasil. Educ Soc. 2011;32(117):1115-21. 
7. Programa das Nações Unidas para o Desenvolvimento. Instituto de Pesquisa Econômica Aplicada. Instituto Brasileiro de Geografia e Estatística. Fundação João Pinheiro. Atlas do desenvolvimento humano no Brasil: índice de desenvolvimento humano municipal. Brasília: PNUD; IPEA; IBGE; FJP; 2003.

8. Rodrigues PC. Bioestatística. 3a edição. Niterói: Universidade Federal Fluminense; 2002.

9. Nahas MV, Rabacow FM, Pereira SV, Borgatto AF. Reprodutibilidade de uma escala para avaliar a percepção dos trabalhadores quanto ao ambiente e às condições de trabalho. Rev Bras Saúde Ocup. 2009;34(120):179-83.

10. Fleck MPA, Louzada S, Xavier M, Chachamovich E, Vieira G, Santos L, et al. Aplicação da versão em português do instrumento de avaliação de qualidade de vida da Organização Mundial da Saúde (WHOQOL-100). Rev Saúde Pública. 1999;33(2):198-205.

11. Fleck MPA, Louzada S, Xavier M, Chachamovich E, Vieira G, Santos L, et al. Aplicação da versão em português do instrumento abreviado da qualidade de vida "WHOQOL-BREF". Rev Saúde Pública. 2000;34(2):178-83.

12. Penteado RZ, Pereira IMTB. Qualidade de vida e saúde vocal de professores. Rev Saúde Pública. 2007;41(2):236-43.

13. Nardi EL, Schneider MP. Condições de trabalho docente: novas tessituras das políticas de avaliação para a qualidade. Educação. 2014;39(1):215-28.

14. Alves T, Pinto JMR. Remuneração e características do trabalho docente no Brasil: um aporte. Cad Pesqui. 2011;41(143):606-39.
15. Marchiori F, Barros MEB, Oliveira SP. Atividade de trabalho e saúde dos professores: o programa de formação como estratégia de intervenção nas escolas. Trab Educ Saúde. 2005;3:143-70.

16. Bauer J, Stamm A, Virnich K, Wissing K, Muller U, Wirsching M. Correlation between burnout syndrome and psychological and psychosomatic symptoms among teachers. Int Arch Occup Health. 2006;79:199-204.

17. Maring EF, Koblinsky SA. Teachers' challenges, strategies, and support needs in schools affected by community violence: a qualitative study. J Sch Health. 2013;83:379-88.

18. Gonçalves CGO, Penteado RZ, Silvério KCA. Fonoaudiologia e saúde do trabalhador: a questão da saúde vocal do professor. Saude Rev. 2005;7(15):45-51.

19. Grillo MHMM, Penteado RZ. Impacto da voz na qualidade de vida de professore(a)s do ensino fundamental. Pró-Fono R Atual Cient. 2005;17(3):321-30.

20. Guglielmi RS, Tatrow K. Occupational stress, burnout, and health in teachers: a methodological and theoretical analysis. Rev Educ Res. 1998;68(1):61-9.

21. Hultell D, Melin B, Gustavsson JP. Getting personal with teacher burnout: a longitudinal study on the development of burnout using a person-based approach. Teaching and Teacher Education. 2013;32:75-86.

Recebido em: 28/01/2014

Aprovado em: 02/10/2013 